\title{
Una vida más allá de las fronteras: Benedict Anderson (1936-2015) ${ }^{1}$
}

\section{A life beyond borders: Benedict Anderson (1936-2015)}

Jorge Bayona ${ }^{2}$

University of Washington (Seattle)

\section{Resumen}

Esta nota es un breve resumen de la vida y obra de Benedict Anderson, uno de los preeminentes especialistas en el sudeste asiático, cuyas contribuciones al estudio de Indonesia, Tailandia y Filipinas siguen influyendo en nuevas generaciones de académicos. $\mathrm{Al}$ conocer algunos de los factores claves de su vida y obra académica - tales como su crianza multicultural, dominio de múltiples lenguas y vigoroso compromiso con los principales acontecimientos políticos que ocurrieron durante su vida - podremos comprender cómo estos dieron pie a la aparición de un libro como Comunidades imaginadas.

Palabras clave: Benedict Anderson, Sudeste asiático, Indonesia, Tailandia, Filipinas

1 Las traducciones del inglés y del indonesio al español son del autor.

2 Candidato doctoral en dicha universidad. Especialista en historia del Sudeste Asiático e historia transpacífica. .Correo electrónico: jbayona@uw.edu 


\begin{abstract}
This is a brief overview of Benedict Anderson's life and work. As one of the world's preeminent Southeast Asianists, his contributions to the study of Indonesia, Thailand, and the Philippines continue to influence newer generations of scholars. Knowledge of some of the key factors of his life and intellectual work - such as his multicultural upbringing, mastery of multiple languages, and vigorous engagement with the major political events of his lifetime- we may understand how a book such as Imagined Communities came about.
\end{abstract}

Key words: Benedict Anderson, Southeast Asian studies, Indonesia, Thailand, Philippines

$$
* * *
$$

En diciembre de 2015, a la edad de 79 años, falleció Benedict Anderson en la isla de Java, Indonesia. Lejos de que el lugar de defunción del autor de Comunidades imaginadas haya sido producto de la casualidad, refleja una vida dedicada al estudio del sudeste asiático, hecho que a veces es pasado por alto en la comunidad académica más extendida. Se cuenta que durante sus últimos años de vida, a sus charlas en universidades alrededor del mundo asistía un público entusiasta convencido de que hablaría del nacionalismo. En lugar de

272 ello, Anderson a veces disertaba sobre temas como su último libro, The fate of rural hell: asceticism and desire in Buddhist Thailand, en el cual describe y reflexiona sobre el significado de un ecléctico templo budista en Tailandia. Lo que más llamó la atención de Anderson fue una serie de esculturas que representaban el infierno donde terminarían los pecadores budistas (Anderson 2012: 11-33, 60-80). Entre las imágenes que mostraba en sus conferencias se podía ver a los peca- 
dores con los genitales al aire, sufriendo toda una serie de creativos tormentos. No era exactamente lo que la audiencia se esperaba de un académico al cual imaginaban únicamente como un adusto experto en el tema del nacionalismo, pero que en realidad fue un hombre jovial y ocurrente ${ }^{3}$, que siempre se consideró, sobre todo, un especialista de área (Anderson 2016: 149).

No obstante, la tendencia a enfocarse exclusivamente en tan solo una fracción de su obra se manifiesta muy claramente en nuestro medio académico. Una rápida búsqueda en los catálogos en línea de algunas de las más importantes bibliotecas universitarias del Perú revela que de todos los libros escritos por uno de los académicos más influyentes del siglo XX y XXI, solo se encuentra disponible uno: el muy conocido Comunidades Imaginadas, que es precisamente el único de su extensa obra que tiene un importante énfasis en occidente. Cabe aclarar que Anderson estuvo muy lejos de ser una suerte de one-hit wonder: la importancia de sus estudios sobre Indonesia, Tailandia y Filipinas fue tal que ganó premios como el Award for Distinguished Contributions to Asian Studies de 1998, otorgado por la Association for Asian Studies de Estados Unidos. Al mantenernos de espaldas a toda esta producción intelectual, da la impresión de que estuviésemos actuando bajo la errada presunción -básicamente eurocéntrica- de que el mundo cognoscible se limitara a América y a Europa occidental, y que el resto del mundo careciera de relevancia o interés. Una revisión de la obra de Anderson nos permitirá comprender la importancia que tiene el ofrecer cursos y estudios sobre todas las regiones del mundo en

3 Comunicación personal de la Dra. Laurie Sears, University of Washington. 
lugar de enfocarse en solo una porción. Esta posibilidad fue fundamental para que surgiera un intelectual con el perfil de Benedict Anderson.

En sus recientemente publicadas memorias intelectuales, Anderson comenta un tropo cultural que me parece pertinente. Señala que tanto en el tailandés como en el indonesio existe la figura de una rana que vive atrapada bajo la cáscara de un coco partido y llega a creer que esa es la extensión total del universo. A lo largo de su texto -y de manera explícita al final de este- Anderson exhorta a todas las ranas del mundo a que se liberen de sus cocos y observen los horizontes de un mundo más amplio (Anderson 2016: 26, 200). En cierto sentido, esa es la intención de esta nota necrológica: echar luces sobre una vida y obra importantísima que en algunos círculos académicos se circunscribe injustamente a Comunidades imaginadas. Con ello en mente, quizá el título de sus memorias publicadas póstumamente - que se podría traducir como una vida más allá de las fronteras - nos pueda brindar un hilo conductor para entender la vida y obra de este intelectual. En lugar de hacer un recorrido cronológico de su vida y obra, me enfocaré en las fronteras que traspasó Benedict Anderson: geográficas, lingüísticas y aquellas que dividen las disciplinas académicas entre sí y a la academia del activismo político.

\section{Traspasando fronteras geográficas y lingüísticas}

Anderson cruzó fronteras geográficas y culturales desde muy temprano en su vida. Nació en Yunnan (China) en 1936 en medio de un hogar multicultural: su padre era irlandés, su madre, inglesa, y la empleada doméstica que ayudó a criarlo era 
vietnamita. En 1941, su familia partió de China con la intención de trasladarse a Irlanda, pero la Segunda Guerra Mundial impidió que pudieran cruzar el Atlántico, de manera que pasaron varios años en los estados de California y Colorado. Al terminar la guerra, su familia finalmente pudo regresar a Irlanda y desde allí partió para estudiar en Inglaterra. Muchos años después comentaría cómo el haber vivido en tantos países diferentes durante sus años formativos le llevaría a no sentir apego por un solo lugar, sino por varios, lo cual a su vez le permitiría después relacionarse profundamente con Indonesia, Tailandia y Filipinas (Anderson 2016: 25).

Durante sus años en Inglaterra se mantuvo en contacto con culturas alejadas cronológicamente de la suya. Adquirió un profundo afecto por los estudios clásicos y perfeccionó el latín durante sus años en Eton y, tras un breve paso por la economía, regresó a aquel campo cuando estudió el pregrado en la Universidad de Cambridge. Pero fue en la Universidad de Cornell -donde estudió ciencia política- que entró en contacto con la región a la que le dedicaría el resto de su vida: el sudeste asiático. Bajo la dirección del politólogo indonesista George Kahin, se interesó por el proceso de independencia de Indonesia y decidió escribir su tesis de doctorado sobre este tema. Fue así que se introdujo al relativamente nuevo campo de los estudios del sudeste asiático, que en buena medida surgió como área propia luego de la Segunda Guerra Mundial y las tensiones de la Guerra Fría. Y cuando las circunstancias lo hicieron necesario, como veremos más adelante, cruzó fronteras y se convirtió en un importante investigador de Tailandia y de Filipinas.

Debido a los grandes desafíos lingüísticos que presenta el estudio del sudeste asiático, no debemos subestimar el es- 
fuerzo necesario para esta empresa. A diferencia de América Latina, donde un hablante nativo del castellano no necesita aprender ninguna nueva lengua para volverse especialista en cualquier otro de los más de veinte países que la usan, en esta parte de Asia cualquier estudio comparativo o traslado de enfoque geográfico requiere aprender por lo menos una o dos lenguas nuevas; por lo general, una nativa y una colonial. No obstante, estas fronteras lingüísticas no fueron impedimento para Anderson. Para su trabajo de campo en Java aprendió indonesio en Cornell, a lo cual después añadió el neerlandés, por medio de un diccionario y la lectura del texto clásico Javaanse Volksvertoningen del académico neerlandés Theodoor Pigeaud, lo cual le fue posible porque ya sabía algo de alemán. A estas dos lenguas, le sumó el estudio del javanés mientras hacía su trabajo de campo (Anderson 2016: 61, 68-70). En el caso de Tailandia, a los 40 años pasó un año sabático en el país y se empapó de la cultura e historia local y aprendió la lengua (Anderson 2016: 90). Finalmente, cuando decidió dedicarse a investigar las Filipinas, no solo aprendió tagalo en Cornell a los 51 años, sino que aplicó al castellano la técnica que había usado anteriormente para el neerlandés: se dedicó a descifrar la novela clásica Noli me tangere del intelectual filipino José Rizal, armado con un diccionario y su conocimiento previo del latín (Anderson 2016: 99-100; 1998: 2). A las seis lenguas que aprendió a lo largo de sus

276 investigaciones, hay que sumar los ya citados alemán y latín, más el francés ${ }^{4}$, por lo menos algo de ruso y, evidentemente, el inglés, es decir un total de por lo menos once lenguas, varias aprendidas en la adultez.

4 El cual podemos ver utilizado numerosas veces en su libro Under three flags: anarchism and the anti-colonial imagination (2005). 
Una anécdota podría ilustrar el grado de familiaridad que llegó a adquirir Anderson con la lengua indonesia. Frustrado al ser tratado como tuan - equivalente a 'amo' o 'Señor' con mayúscula- por el mero hecho de ser blanco, pese a ser tan solo un estudiante de posgrado, decidió encontrar un término más adecuado. $\mathrm{Al}$ percatarse de que su tono de piel se acercaba al de los carabaos albinos, Anderson les indicó a sus amigos indonesios que usaran ese término para referirse a él y a aquellos que se le parecieran: bulé (Anderson 2016: 77). Esta ocurrente palabra se ha incorporado completamente al habla común en Indonesia a lo largo del último medio siglo. Es como si Benedict Anderson hubiera inventado el equivalente a la palabra gringo. Fue tanta su fluidez y sutilidad en dicha lengua que incluso publicaba artículos satíricos en dicha lengua en la prensa de Jakarta.

Este constante cruce de fronteras geográficas y lingüísticas fue sumamente productivo para su trabajo. Siempre mantuvo presente un pasaje de Noli me tangere, en que el protagonista observa el dilapidado jardín botánico de Manila tras regresar de una larga estancia en Europa y el narrador comenta que "el demonio de las comparaciones le puso delante los jardines botánicos de Europa” (Rizal 1886: 43). Del mismo modo en que Rizal y su protagonista se vieron obligados a hacer comparaciones entre las distintas realidades con las que se habían familiarizado, Anderson también sintió este impulso e hizo de este una constante en su obra académica, como se ve claramente en Comunidades imaginadas. Es más, tomó prestado el término de Rizal como título para su libro The spectre of comparison: nationalism, Southeast Asia, and the world'.

5 Aunque después admitió que el uso de spectre fue un error de traducción suyo (Anderson 2005: 32). 
Este es un libro particularmente interesante que muestra la estrategia comparativa de Anderson, la cual considera un mecanismo discursivo y no una metodología. Por ejemplo, incluye ensayos en los que compara aspectos como las elecciones y el destino de los partidos comunistas en sus tres países predilectos: Indonesia, Tailandia y Filipinas. Al analizar el efecto de la crisis financiera de 1997 y los conceptos de mayorías y minorías, incluye a Birmania/Myanmar y también a Malasia. En el ensayo "Sauve qui peut" pronosticó correctamente que la dictadura de Soeharto estaba ya herida de muerte, mientras que en "Majorities and minorities" explica que las mayorías y minorías fueron creadas por la experiencia colonial y cómo estas se vieron utilizadas tras la independencia. La mirada de Anderson sobre el mundo es tan amplia que incluso incluye un ensayo sobre el Perú, "El malhadado país", en el que comenta las implicancias de una novela de Mario Vargas Llosa y lo que le sugiere en comparación con novelistas del sudeste asiático como Pramoedya Ananta Toer o José Rizal (Anderson 1998: 333-359) ${ }^{6}$.

\section{Traspasando fronteras entre disciplinas}

La formación académica de Benedict Anderson en Cornell fue propiamente en el campo de la ciencia política, en ese entonces conocido como "gobierno". Comenta, sin embargo, que en la época en que estudió las fronteras entre las disciplinas no estaban tan marcadas como lo llegarían a estar después y que la presión por llevar seminarios solo dentro de su de-

6 Este es quizá uno de los pocos trabajos de Anderson, aparte de Comunidades imaginadas, que ha sido comentado por algún intelectual peruano. $\mathrm{Al}$ respecto, véase Faverón 2002. 
partamento era casi inexistente. Esto le permitió llevar asignaturas en diversos programas, lo cual tuvo un notable efecto sobre su producción académica. Por tomar como ejemplo a Comunidades imaginadas, el mismo Anderson no sabe en qué categoría colocarlo: "Definitivamente, no pertenece a la historia, puesto que no se basa en fuentes de archivo ni primarias. ¿Ciencia política? Tan solo uno o dos libros de ciencia política son mencionados en la bibliografía" (Anderson 2016: 157). Esta característica de no mantenerse encuadrado dentro de una sola disciplina y más bien tomar ideas útiles provenientes de muchas otras se manifiesta a lo largo de su obra. Con la posible excepción de tres ensayos en Language and power: exploring political cultures in Indonesia (Anderson 1990: 17-120), las disquisiciones metodológicas y teóricas tan habituales en la producción académica suelen estar más bien ausentes en muchos de sus libros, los cuales se enfocan en la apertura del conocimiento respecto de las sociedades que estudia y no en la demostración o refutación de corrientes intelectuales en boga en el momento de su publicación.

Anderson dio una temprana muestra de este deseo de traspasar las fronteras tradicionales de las disciplinas en su monografía Mythology and the tolerance of the Javanese, en la que recurrió al teatro y la mitología, y no a la ciencia política, para explicar la tolerancia religiosa y política que vio en Java de la década de 1960. A partir de la estética del milenario teatro de sombras - wayang-, Anderson arguye que al presentar una serie de arquetipos muy variados, pero todos igualmente admirables, provenientes del Mababharata y del Ramayana, se instiló en el pueblo javanés la idea de que había muchas formas diferentes de ser virtuoso (Anderson 1965). Asimismo, en Java in a time of revolution: occupation and resistance, 19441946, basado en su tesis de doctorado, en lugar de realizar 
un estudio que correspondiera a los criterios usados en la ciencia política de aquel entonces, es decir, temas como las lealtades, posiciones ideológicas y redes de contactos de personajes como Soekarno, Hatta, Sjahrir o Tan Melaka, empezó su libro dedicando espacio a las condiciones de vida de la pemuda, es decir, la juventud javanesa. En un par de capítulos que parecen más propiamente etnológicos o antropológicos explica la condición liminar de la pemuda: están en una fase intermedia de la vida, atrapados entre la niñez y la adultez, durante la cual abandonan el hogar familiar para estudiar con maestros islámicos, de artes marciales o-a partir de la época de la Política Ética (1901-1942) y en números limitados- en escuelas de estilo occidental. Alejados de los lazos familiares y en recintos exclusivamente masculinos, estos jóvenes vivían un breve momento de relativa libertad para imaginar un futuro completamente diferente de aquel en el que se encontraban, un futuro mesiánico (Anderson 1972: 1-34). Estas fueron las condiciones que posibilitaron que los militares japoneses pudieran reclutarlos como fuerzas de autodefensa en caso de que los aliados intentaran retomar Java por medio de las armas y ayuda a explicar el entusiasmo con el que se acometieron a crear una nueva sociedad. Asimismo, esta visión etnográfica nos permite comprender la actitud independiente e intransigente que asumieron tras la rendición japonesa (Anderson 1972).

Si su trabajo en torno a Indonesia pareciera estar a mitad de camino entre la ciencia política y la antropología, sus estudios sobre las Filipinas lo estarían entre la historia y la literatura. Esto se puede ver desde su ensayo clásico "The First Filipino" en torno al mundo cultural del nacionalista e intelectual filipino José Rizal. A partir de las novelas Noli me tangere y El filibusterismo, Anderson comenta la condición pionera de 
Rizal a la hora de jugar con la frontera entre la realidad y la ficción para arremeter contra los aspectos más sórdidos del colonialismo español, así como las complejidades inherentes a la elección del castellano en lugar del tagalo como la lengua en que escribiría. De este modo, Anderson propone que Rizal estaba de cierto modo dirigiéndose a un público universal que comprendía tanto a sus amigos como a sus enemigos, en las Filipinas o fuera de ellas (Anderson 1998: 227-234). Esta productiva aproximación se ve ampliada y profundizada en su libro Under three flags: anarchism and the anti-colonial imagination. En este texto, la mirada de Anderson se desplaza vertiginosamente desde las Filipinas a Francia, o desde el Caribe a España. Busca situar la producción académica y novelística de José Rizal e Isabelo de los Reyes, otro importante intelectual filipino, en un contexto de febril actividad intelectual e ideológica a nivel mundial. Este contexto les serviría a ambos autores, desde sus respectivas empresas intelectuales, ayudar a socavar el colonialismo español. Lejos de querer ser aceptados como iguales a los peninsulares, lo que había buscado el movimiento de propaganda de Marcelo del Pilar y otros, estos autores estaban expandiendo sus horizontes intelectuales para situarse más allá de una España a la que consideraban atrasada respecto del resto de Europa. De este modo, los estudios de folclore realizados por De los Reyes eran un intento de circunvalar a la metrópoli y poner a las Filipinas a la altura de Inglaterra y Alemania, donde dicha disciplina cobraba fuerza. Asimismo, las novelas de Rizal, lejos de ser textos meramente provinciales, son el resultado de la fermentación de las tendencias vanguardistas y de las letras europeas, así como de las actividades y proselitismo anarquista que estuvieron en boga durante los largos periplos de Rizal por Europa. Anderson arguye convincentemente que estos son personajes de interés universal. 
Quizás en el caso de Tailandia es donde más claramente se pueda ver el efecto liberador que tuvo en su trabajo el haber pasado al retiro en 2001 (Anderson 2016: 163-166). Ya desde la década de 1980 se sentía un tanto sofocado por la presión disciplinaria de la ciencia política, mientras que sus intereses se inclinaban por explorar las relaciones entre la sociedad y la ficción. Por ejemplo, cuenta que al publicar una colección de ficción tailandesa titulada In the Mirror (Mendiones y Anderson 1985) sus colegas de dicha disciplina le increparon que "después de escribir un libro de alta teoría como Comunidades imaginadas, ¿por qué rayos andas traduciendo cuentos tailandeses?" (Anderson 2014: 94). No obstante, en 1990 publicó "Murder and progress in modern Siam", ensayo en el cual a partir de una película sobre sicarios hacía comentarios interesantes y contraintuitivos sobre la apertura democrática tailandesa (Anderson 2014: 101-115). En cualquier caso, la jubilación le permitió adentrarse en temas no solo de literatura, sino también de cinematografía y antropología. Sus ensayos "The strange story of a strange beast: receptions in Thailand of Apichatpong Weerasethakul's Sat Pralaat' y "Mundane history/Jao nok krajok" (ambos originalmente publicados en tailandés) denotan una sutil comprensión de la sociedad e historia tailandesas. En el caso de Sat Pralaat, Anderson argumenta convincentemente que la trama mágica y homosexual de la película apela a tradiciones profundamente enraizadas en la cultura rural tailandesas y que no se trata de un filme europeizado, como lo entendieron las élites educadas de Bangkok (Anderson 2014: 131-146).

\section{Traspasando la frontera entre la academia y el activis- mo político}

En sus memorias, Benedict Anderson comenta que "lo que diferencia a los especialistas de área de los académicos enfo- 
cados en sus disciplinas es el apego emocional que sentimos por los lugares y pueblos que estudiamos" (Anderson 2016: 164). A lo largo de su carrera, Anderson buscó no aislarse en una torre de marfil y más bien mantenerse en contacto con las realidades de los pueblos que estudiaba, y conservó esa actitud incluso cuando ello le significó enormes perjuicios. Firmemente en contra de las dictaduras en Indonesia, Tailandia y Filipinas, así como la guerra de Vietnam y la invasión indonesia de Timor Leste, para él era incomprensible que un académico no tomara partido por las poblaciones vulnerables.

Para empezar, la causa detrás de la migración de Anderson de los estudios indonesios a los tailandeses no fue el aburrimiento o diletantismo, sino el haber sido expulsado de Indonesia como consecuencia de su actitud frente al golpe de Estado de octubre de 1965. En los meses posteriores a la toma del poder por parte de Soeharto, las matanzas colectivas promovidas por el ejército indonesio y las fuerzas derechistas del país cobraron las vidas de por lo menos medio millón de personas, muchas de las cuales eran miembros del partido comunista de Indonesia, el más grande del mundo fuera del bloque sino-soviético. Ante esta situación, Benedict Anderson y la indonesista Ruth McVey se dedicaron a investigar y redactar un informe sobre dichos acontecimientos, que fue conocido como el 'Cornell paper'. En este desmontaban el discurso justificatorio que había promovido el ejército y las fuerzas conservadoras. El "golpe de izquierda" que había precipitado la reacción de Soeharto no había sido realizado por el partido comunista, el cual ni siquiera había estado al tanto de que se realizaría, sino por oficiales de mando medio del ejército (Anderson y McVey 1971). Este informe le ganó la enemistad de Soeharto, quien prohibió el regreso de 
Anderson. Este no pudo regresar al país sino cuando hubo caído el régimen en 1998.

Pese a haber perdido el acceso al país, Anderson jamás abandonó por completo su interés académico por Indonesia. Interpreting Indonesian politics, editado por él y Audrey Kahin en 1982 compiló una serie de artículos de varios prominentes indonesistas. Su ensayo "Perspective and method in American research on Indonesia" fue una descripción y crítica de las perspectivas utilizadas por los académicos estadounidenses que estudiaron Indonesia después de la Segunda Guerra Mundial y hasta la guerra de Vietnam. Según Anderson, en el periodo 1955-1965, dichos académicos privilegiaron una visión modernizadora del país mediante la cual los autonomistas indonesios fueron representados como agitadores comunistas que impedían el progreso, pero que la crisis de Vietnam presentaba una oportunidad para cambiar el paradigma (Anderson y Kahin 1982: 69-83). El haber sido expulsado de Indonesia también le permitió escribir "Gravel in Jakarta's shoes", ensayo en el cual Anderson critica ferozmente la invasión indonesia (en connivencia con políticos estadounidenses) de Timor Leste y las prácticas brutales usadas en la guerra de 1975-1978. En esta empresa imperial, los indonesios cometieron todo tipo de atrocidades que resultaron en la muerte de centenares de miles de timorenses, cuya historia

284 Anderson expone y denuncia (1998: 131-138). Tras el final de su exilio, publicó en indonesio el ensayo "Tentang matinya para jenderal" ("En torno a la muerte de los generales") en Yogyakarta, en el cual a partir del informe médico de la necropsia de los generales muertos durante el primer golpe de Estado de 1965 concluye que no fueron torturados antes de morir (Van Langenberg, Anderson y Dale-Scott 1999: $52-$ 
53), y editó Violence and the state in Suharto's Indonesia ${ }^{7}$, el cual incluye artículos de una nueva generación de indonesistas que pudieron estudiar el legado de este régimen autoritario (Anderson 2001).

Lo que atrajo a Anderson hacia Tailandia fue también una coyuntura política: en 1973 un movimiento estudiantil desembocó en la caída del régimen militar, con lo cual se dio una suerte de primavera democrática hasta 1976. Él no habría dado este paso antes de este giro; comenta que en el contexto de la intervención estadounidense en Vietnam él, como todos los especialistas en Vietnam e Indonesia, se encontraba firmemente en contra, mientras que quienes estudiaban Tailandia la apoyaban (Anderson 2016: 54-55). Ahora bien, uno podría pensar que una vez expulsado de Indonesia Benedict Anderson se cuidaría de no volver a criticar al gobierno del país que estudiaba. Sin embargo, Anderson no dejó de tener una mirada crítica respecto de lo que pasaba en dicho país. Poco después del golpe de Estado de 1976 publicó "Withdrawal symptoms: social and cultural aspects of the October 6 coup" donde critica la retoma del poder por parte del ejército y las masacres de grandes números de estudiantes universitarios en el proceso (Anderson 2014: 47-76). Esta actitud es particularmente notable tomando en cuenta que Tailandia tiene unas de las leyes de lèse-majesté más drásticas del mundo, las cuales son usadas de manera muy flexible respecto de todo aquel que cuestione el orden impuesto por las fuerzas conservadoras ${ }^{8}$. Manteniéndose firme en sus

7 El título del libro utiliza la ortografía alterna "Suharto".

8 Asimismo, en "Two unsendable letters", Benedict Anderson critica la actitud tailandesa de aceptar las disculpas birmanas por la destrucción de la antigua capital de Ayutthaya, pero la ausencia de disculpas tailandesas 
principios, Anderson recordaba con amargura cómo los tailandistas estadounidenses se rehusaron a firmar una carta de condena al golpe de Estado de 1976 y los indonesistas evitaron manifestarse en contra de la invasión de Timor Leste, por temor a que se les prohibiera el acceso a los países que investigaban, como le sucedió a él (Anderson 2016: 94).

Su interés por las Filipinas también se vio marcado por su rechazo a proyectos políticos autoritarios. A inicios de la década de 1970 advirtió en Filipinas que Ferdinand Marcos estaba en camino de establecer una dictadura como lo había hecho Soeharto en Indonesia algunos años antes. El tiempo le dio la razón y en 1972 Marcos instauró su régimen de ley marcial bajo el cual destruyó cualquier huella de oposición. Fue con la caída del dictador en 1986 que finalmente decidió dejar momentáneamente su estudio de Tailandia para dedicarse a las Filipinas (Anderson 2016: 97-100). Anderson reconoce que le tiene cierta deuda al dictador Soeharto puesto que, de no haber sido expulsado por él, jamás habría estudiado ningún país aparte de Indonesia y, por ende, probablemente jamás habría escrito Comunidades imaginadas (Anderson 2016: 55). En el caso de Anderson vemos cómo las inclinaciones políticas de un intelectual pueden tener, si bien de manera algo fortuita, muy fecundos resultados para el mundo de la academia.

por el saqueo de Angkor en el siglo XV, o la anexión de Camboya en el siglo XIX, entre otros. Imaginen lo que significaría que un académico extranjero especializado en Chile reclamara que pidieran disculpas por la guerra del Pacífico, o que uno especializado en el Perú hiciera un llamado para que pidieran disculpas por la invasión a Ecuador en 1941. 


\section{El impacto de la obra de Benedict Anderson}

Ciertamente, no se puede subestimar el impacto que tuvo Comunidades imaginadas. Sería imposible hacer un listado de todos los textos que dialogan con el concepto propuesto por Anderson, puesto que estos se extienden desde América Latina, como en el caso de Beyond imagined communities: Reading and writing the nation in nineteenth-century Latin America (Castro-Klarén y Chasteen 2003) hasta el Mediterráneo oriental, como en el caso de Imagined communities in Greece and Turkey: trauma and the population exchanges under Atatürk (Bedlek 2016), por citar solo dos ejemplos. Ha sido también el tema de la compilación Grounds of comparison: around the work of Benedict Anderson, en la que literatos, antropólogos e historiadores comentaron sus ideas y fueron respondidos por el mismo Anderson (Culler y Cheah 2003). Prácticamente, a nadie que esté leyendo esta nota tiene que recordársele la importancia de aquel famoso texto. Como mi objetivo ha sido más bien abrir nuestros horizontes al resto de la muy importante obra de Benedict Anderson, haré una recensión del valor de sus aportes en el campo de los estudios de Indonesia, Tailandia y Filipinas. Como veremos, el valor de la obra de Anderson como especialista en el sudeste asiático no se limita solo a solo ese campo, donde es muy valioso, sino que fue la condición que posibilitó la formulación de aportes intelectuales de relevancia general.

El consenso entre los académicos que estudian el sudeste asiático es que Benedict Anderson fue una figura especialmente importante y ha ameritado textos dedicados a él por numerosos académicos. Este es el caso de Southeast Asia over three generations: essays presented to Benedict R. O'G. Anderson. En su introducción, al hablar de las tempranas obras de An- 
derson enfocadas en el sudeste asiático, James Siegel dice "En lugar de desaparecer, siguen siendo sugerentes. Uno no puede clasificar estos estudios o decir cuáles son sus puntos centrales. Más bien abren un mundo. Al releerlos, uno reconoce verdades, aun cuando en algunos casos las condiciones de dichas verdades hayan cambiado radicalmente" (Siegel y Kahin 2003: 8). Asimismo, el académico indonesio Ariel Heryanto ha dicho que "la obra de Ben disfruta del alto respeto que se merece entre muchos académicos que dirigen y están formalmente afiliados tanto a los estudios de Asia como a disciplinas tradicionales" (Heryanto 2016: 163). Los autores de $A$ history of Thailand, Pasuk Phongpaichit y Christopher Baker, comentan "Sus cuatro grandes ensayos sobre la política tailandesa tuvieron una enorme influencia en nuestro trabajo" (Phongpaichit y Baker 2016: 166). El especialista de Tailandia James Ockey señala que los artículos posteriores de Anderson, a pesar de no ser tan conocidos por la comunidad académica por haber sido publicados en tailandés y orientados a un público no académico, "siguen ampliando las fronteras de nuestra comprensión de la política tailandesa, y especialmente la relación de la política con la condición del tailandés y la cultura siamesa en sentido más general" (Ockey 2015: 309). Al comentar cómo Anderson contextualiza a José Rizal como figura universal, el historiador filipino Vicente Rafael asevera que "su grandeza como 288 académico y profesor yacía en su habilidad para fisgonear bajo capas de engaños y medias-verdades ocultas por los hábitos de ociosidad intelectual y cobardía moral" (Rafael 2016: 142).

Una forma alternativa de evaluar la influencia de la obra de Benedict Anderson es verificar si es tomado en cuenta en los libros de síntesis histórica sobre los países estudiados por 
él. Podemos constatar que varios de los libros de texto más recientes lo incluyen entre sus fuentes, como se puede ver en los casos de Indonesia: peoples and histories (Taylor 2003: 405, 407, 409), State and society in the Philippines (Abinales y Amoroso 2005: 314) y - para la región en su conjunto- $A$ bistory of Southeast Asia: critical crossroads (Reid 2015: 434) y Southeast Asia: past and present (SarDesai 2003: 425). En el género de los readers, aparece un texto suyo sobre la lengua indonesia en el Indonesia reader: history, culture, politics (Hellwig y Tagliacozzo 2009: 271-274) y se le incluye en la bibliografía del Southeast Asia in the twentieth century: a reader (Christie 1998: 327, 328). En el caso de Tailandia, quizá por el hecho de que parte de su obra tardía se publicó solo en tailandés, no se le encuentra tan presente al nivel de síntesis. Es posible que esto cambie con la reciente recopilación de sus diversos ensayos (Anderson 2014). Asimismo, tomando en cuenta que buena parte de estos trabajos (salvo el de Reid) se publicaron antes que los trabajos tardíos de Anderson, cabe esperar que la influencia de estos siga ampliando la estela de este académico en el campo de los estudios del sudeste asiático.

Por supuesto, cabe comentar algo de Comunidades Imaginadas y su relación con los estudios del sudeste asiático. Anderson comenta que este "extraño libro" - así lo describe él- fue escrito como respuesta a una generación de historiadores británicos con quienes se familiarizó por intermedio de su hermano Perry Anderson. Estos enfocaban el nacionalismo como un fenómeno propiamente europeo, el cual fue posteriormente imitado por el resto del mundo (Anderson 2016: 123-125). Fue necesaria la intervención de un ecléctico políglota especializado en varios países del sudeste asiático para desvelar el etnocentrismo del que pecaban aquellos académicos europeístas. Sin duda, habrá habido aquellos que al leer 
Comunidades imaginadas se hayan sentido un tanto desconcertados por las numerosas referencias a novelistas filipinos, reyes siameses o dictadores indonesios en un texto que entendían como correspondiente al género de la historia universal, género en el cual se intuye que los casos de estudio deberían salir principalmente de Europa. La misma inspiración para el libro, la guerra sino-vietnamita de 1979, está fuera del mundo mental de mucho de sus lectores. Pero ese es precisamente el punto: el estudio de varios países del sudeste asiático por Benedict Anderson no es meramente anecdótico, en realidad, solo alguien con una perspectiva comparativa tan amplia como la proporcionada por el estudio de esos países podría haber escrito un libro como Comunidades imaginadas.

Esta es quizá la lección más valiosa que nos deja la vida y obra de Benedict Anderson. No hay programa o malla curricular fija que podría formar a un intelectual de este tipo; fue el resultado de no solo una vida de experiencias multiculturales tempranas, sino que el contexto universitario y académico en el que estudió, enseño e investigó acogió una gran diversidad de regiones y temáticas de estudio, además de brindar el apoyo necesario con bibliotecas, docentes y programas de lenguas especializados. Recae sobre nuestra academia liberarse de la proverbial cáscara de coco y desechar la premisa de que lo único que le corresponde estudiar a un peruano

290 es el Perú y que, por ende, lo único pertinente del resto del mundo vendría a ser la tradición occidental. Si alguna lección nos hemos de llevar de la obra de Benedict Anderson es que la ampliación de la oferta de temas de estudio para nuestras futuras generaciones de académicos puede tener resultados muy productivos y completamente impredecibles.

Recibido: 28 de junio del 2016

Aprobado: 18 de enero del 2017 


\section{Bibliografía}

ABINALES, Patricio y Donna AMOROSO

2005 State and society in the Philippines. Lanham: Rowman and Littlefield.

ANDERSON, Benedict

$2016 \quad$ A life beyond boundaries. London and New York: Verso.

2014 Exploration and irony in studies of Siam over forty years. Ithaca: Cornell Southeast Asia Program Publications.

2012 The fate of rural hell: asceticism and desire in Buddhist Thailand. London, New York and Calcutta: Seagull Books.

2005 Under three flags: anarchism and the anti-colonial imagination. London and Nueva York: Verso.

2001 Violence and the state in Soeharto's Indonesia. Ithaca: Southeast Asia Program Publications, Cornell University.

1998 The spectre of comparison: nationalism, Southeast Asia, and the world. London and New York: Verso.

1991 Imagined communities: reflections on the origin and spread of nationalism. London and New York: Verso.

1990 Language and power: exploring political cultures in Indonesia. Ithaca: Cornell University Press.

1972 Java in a time of revolution: occupation and resistance, 19441946. Ithaca: Cornell University Press.

1966 "Japan: "the light of Asia". En Josef SILVERSTEIN (editor). Southeast Asia in World War II: four essays. New Haven: Yale University, pp. 13-50. 
Mythology and the tolerance of the Javanese. Ithaca: Southeast Asia Program-Cornell University.

ANDERSON, Benedict y Ruchira MENDIONES (editors)

1985

In the mirror: literature and politics in Siam in the American era. Bangkok: Editions Duang Kamol.

ANDERSON, Benedict y Audrey KAHIN (editors)

1982 Interpreting Indonesian politics: thirteen contributions to the debate. Ithaca: Southeast Asia Program-Cornell University.

ANDERSON, Benedict, Michael VAN LANGENBERG y Peter DALE-SCOTT

1999 Gestapu, matinya para jenderal, dan peran CLA. Yogyakarta: Cermin.

ANDERSON, Benedict y Ruth MCVEY

A preliminary analysis of the October 1, 1965 coup in Indonesia. Ithaca: Modern Indonesia Project, Cornell University.

BAKER, Christopher y Pasuk PHONGPAICHIT

2016 "Benedict Anderson's 'Strange hierarchies' in Thailand." Philippine studies: historical and ethnographic viewpoints. Manila, Volume 64, number 1, pp. 166-178. https://doi.org/10.1353/phs.2016.0000

2005 A history of Thailand. New York: Cambridge University Press.

BEDLEK, Emine Yeşim

2016

Imagined communities in Greece and Turkey: trauma and the population exchanges under Atatürk. London: I.B. Taurus.

CASTRO-KLARÉN, Sara y John Charles CHASTEEN (editors) 2003 Beyond imagined communities: Reading and writing the nation in nineteenth-century Latin America. Baltimore: Johns Hopkins University Press. https://doi.org/10.1353/hir.2005.0043 
CULLER, Jonathan y Pheng CHEAH (editors)

2003 Grounds of comparison: around the work of Benedict Anderson. New York: Routledge.

https://doi.org/10.4324/9780203954362

FAVERÓN, Gustavo

2002 "Comunidades inimaginables: Benedict Anderson, Mario Vargas Llosa, la novela y América Latina." Lexis. Lima, Volume 26, pp. 441-467.

HELLWIG, Tineke y Eric TAGLIACOZZO

2009 The Indonesia reader: history, culture, politics. Durham: Duke University Press. https://doi. org/10.1215/9780822392279

HERYANTO, Ariel

2016 "Benedict Anderson: a great inspiration." Philippine studies: historical and ethnographic viewpoints. Manila, Volumen 64, number 1, pp. 159-166.

https://doi.org/10.1353/phs.2016.0012

OCKEY, James

2015 "Benedict Anderson and Siam Studies." Journal of Southeast Asian Studies. Singapur, Volume 42, number 2, pp. 293-309

https://doi.org/10.1017/s0022463415000090

RAFAEL, Vicente

2016 "Contingency and comparison: recalling Benedict Anderson." Philippine studies: historical and ethnographic viewpoints. Manila, Volume 64, number 1, pp. 135-144. https://doi.org/10.1353/phs.2016.0004

REID, Anthony

2015 A history of Southeast Asia: critical crossroads. Chichester: Wiley Blackwell.

https://doi.org/10.1080/10357823.2016.1202172 
RIZAL, José 1886

Noli me tangere. Berlín: Berliner buchdruckerei-actiengesellschaft. https://doi.org/10.2143/1s.32.3.2033416

SARDESAI, D. R.

2003 Southeast Asia: past and present. Boulder: Westview Press. https://doi.org/10.2307/2760907

SIEGEL, James y Audrey KAHIN (editors)

2003 Southeast Asia over three generations: essays presented to Benedict R. O'G. Anderson. Ithaca: Southeast Asia Program, Cornell University.

https://doi.org/10.1355/cs26-3h

TAYLOR, Jean

2003

Indonesia: peoples and histories. New Haven: Yale University Press.

https://doi.org/10.2307/20033738 